\title{
Synthesis, Characterization and Liquid-Carrying Capacity of Zeolitic Material from Circulating Fluidized Bed Fly Ash
}

\author{
Jay V. Ranoco, JC Justine G. Quintos, and Rizalinda L. de Leon
}

\begin{abstract}
With the growing awareness on the environmental impact of coal-burning, conventional Pulverized Coal-fired (PC) boilers are increasingly being replaced with Circulating Fluidized Bed (CFB) boilers. This shift in technology however, has brought about a new environmental impact - its generation of tons of fly ash that has yet to find utilization. About 7.2 metric tons per year of fly ash is expected to be generated upon completion of the CFB boilers being installed in the country. CFB fly ash, unlike PC fly ash, cannot be directly utilized by the cement and petroleum refining industries due to its unsuitable chemical and physical properties. In order to put value to CFB fly ash, the researchers transformed CFB fly ash into zeolitic materials through fusion with $\mathrm{NaOH}$ at elevated temperatures. The synthesized zeolites underwent confirmatory tests such as Fourier Transform Infrared Spectroscopy (FTIR), Scanning Electron Microscopy (SEM) and X-Ray Diffraction (XRD). IR spectra pattern comparison of commercial and synthesized product showed successful transformation of fly ash into element components that are precursors to zeolites. XRD and SEM analysis showed the transformation of fly ash into an amorphous phase material. Its liquid carrying capacity was determined as a measure of zeolite performance as laundry detergent surfactant carrier.
\end{abstract}

Index Terms - CFB fly ash, zeolitic material, liquid-carrying capacity.

\section{INTRODUCTION}

Burning coal generates about $74 \%$ fly ash [1]. This huge generation of fly ash poses a disposal problem. There have been studies which exploit the composition of this waste material to form valuable products. Coal fly ash is a good source of silica and alumina, about $40-55 \%$ of fly ash is silica $\left(\mathrm{SiO}_{2}\right)$ and about $20-25 \%$ is alumina $\left(\mathrm{Al}_{2} \mathrm{O}_{3}\right)$. As is, fly ash does not exhibit good catalytic properties, attributed to its very low specific surface area with around $1.4 \mathrm{~m}^{2} / \mathrm{g}$ [2].

However, the silicon and aluminum content of fly ash can be used and transformed into something more valuable. Fly ash derived from pulverized coal fired boilers has been utilized as cement substitute or concrete additives. In the Philippines, conventional pulverized coal-fired (PC) boilers are being replaced with cleaner Circulating Fluidized Bed

Manuscript received March 25, 2016, revised August 5, 2016. This work was supported in part by the Unilever Undergraduate Research Grant from the Unilever Philippines and was done at the Fuels, Energy and Thermal Systems Laboratory of the Department of Chemical Engineering, College of Engineering, University of the Philippines, Diliman, Quezon City, MM 1101 PHILIPPINES.

R. L. de Leon is with University of the Philippines, Philippines (e-mail: rizalinda.deleon@coe.upd.edu.ph).
(CFB) boilers for power and steam generation. CFB's main advantages over previous technologies include fuel flexibility and multi-fuel firing capability. CFB generates fewer ${ }^{1}$ sulfur oxides (SOx) and nitrogen oxides (NOx) emissions but still produces fly ash. Because of increasing number of CFBs, CFB fly ash generation is also increasing. This huge amount of waste generation faces a great challenge for the Philippines on where and how to dispose these materials. CFB fly ash, unlike regular coal-fired burner fly ash, cannot be directly utilized by cement industries as additives for cement and road blocking due to their high sulfur content and unsuitable morphology. A total estimate of 7.2M TPY of fly ash is to be generated after the completion of CFB boilers being installed in the Philippines.

Zeolites are microporous materials, primarily used as molecular sieves, ion exchange media, adsorbent and catalyst [3]. They naturally occur in the reaction of volcanic rocks and ash layers with groundwater. Guth and Kessler [4] technically defined zeolites as crystalline solids having a tetrahedral framework composed of $\mathrm{TO}_{4}$ elements $(\mathrm{T}=\mathrm{Si}, \mathrm{Al})$. Each of the oxygen elements in this framework are shared by two $\mathrm{T}$ elements. In the framework also exists cavities and channels in which charge-compensating ions or other molecules are hosted and can be exchanged. The interiors of the zeolite pores are catalytically active [3]. The general formula for zeolite is described by $\mathrm{M}^{\mathrm{i}} \mathrm{M}^{\mathrm{ii}}{ }_{0.5}\left[\left(\mathrm{AlO}_{2}\right)_{\mathrm{x}}\left(\mathrm{SiO}_{2}\right)_{\mathrm{y}}\left(\mathrm{H}_{2} \mathrm{O}\right)_{\mathrm{z}}\right]$, where $\mathrm{M}^{\mathrm{i}}$ and $\mathrm{M}^{\mathrm{ii}}$ are cations with charges (i) and (ii), $\mathrm{x}$ and $\mathrm{y}$ are the oxide numbers and $\mathrm{z}$ is the hydration number.

Since zeolite is a crystalline structure, its synthesis in the laboratory follows the principles of crystallogenesis. The first step in crystal formation is nucleation. This happens when the free energy decrease of creating a new volume exceeds that of the increase in making a new interface. There is a critical radius which, when exceeded, the nucleus will favor growth rather than dissolution. The degree of supersaturation inversely affects the said critical radius. The second step is crystal growth. A newly formed nucleus keeps growing until it reaches thermodynamic equilibrium with the solution. For complex system like zeolites, if the system is far from equilibrium it tends to form intermediate metastable phases before the thermodynamically stable phase (Ostwald's rule). Compared to a supersaturated (out of equilibrium) sugar solution in which an introduction of suitable nucleation sites accelerates the formation of crystals to attain equilibrium, zeolite systems have intermediate metastable phases and will not immediately form stable crystals. One of metastable states is an amorphous gel phase which has a role in the synthesis as described later in this text. 
Guth and Kessler [4] listed three (3) main constituents involved in the synthesis system: source of framework elements, the template, and the mineralizer. For the first constituent, the Silicon and Aluminum will be sourced from the $\mathrm{Si}$ and $\mathrm{Al}$ content of CFB fly ash, in the form of $\mathrm{SiO}_{2}$ and $\mathrm{Al}_{2} \mathrm{O}_{3}$.

The mechanism of zeolite synthesis starts when the T-elements are dissolved together in high concentrations. The high concentrations of these anions, however do not promote crystal growth. Nuclei radii are too small for further development. Ostwald's rule dictates then the formation of metastable gel in the solution, lowering the concentrations of aluminosilicate species in solution and the degree of supersaturation. This gel has enough solubility to provide supersaturation suitable for crystal formation. As soon as the degree of supersaturation of the solution is suitable, zeolites may begin to crystallize. The soluble T-elements bind to the crystal zeolite framework and they are replaced by T-elements detaching from the gel by dissolution. The gel becomes the reservoir of the framework elements until its disappearance.

Much of the reviewed literature investigated as part of the work described the synthesis of zeolitic materials from regular pulverized coal fly ashes. Shigemoto, et al. fused fly ash from Australian and Japanese (Miike) coal (2.2 $\mathrm{SiO}_{2} / \mathrm{Al}_{2} \mathrm{O}_{3}$ ) with hydroxide to produce $\mathrm{Na}-\mathrm{X}$ type zeolites [5]. Ojha, et al. (2004) synthesized X-type zeolites from pulverized coal-fired power plant fly ash (1.89 $\left.\mathrm{SiO}_{2} / \mathrm{Al}_{2} \mathrm{O}_{3}\right)$ [6]. Sutarno, et al. (2007) succeeded in synthesizing faujasite type zeolites and has determined optimum $\mathrm{HCl}$ concentration and $\mathrm{NaOH} / \mathrm{Fly}$ Ash weight ratio for a high $\mathrm{Si} / \mathrm{Al}$ ratio product [7]. Koukouzas, et al. tested and succeeded in investigating the ability of Polish bituminous $\left(1.56 \mathrm{SiO}_{2} / \mathrm{Al}_{2} \mathrm{O}_{3}\right)$ and South African coal (1.4 $\left.\mathrm{SiO}_{2} / \mathrm{Al}_{2} \mathrm{O}_{3}\right) \mathrm{CFB}$ derived fly ash to produce zeolitic products [8].

Experimental factors include solvent, source of T elements, mineralizer, templates, reaction mixture composition, reactant preparation, aging time, seeding, nature of reactor, crystallization temperature, pressure, agitation, and heating rate [4].

This study performed the synthesis and characterization of zeolitic material from Circulating Fluidized Bed fly ash obtained from burning co-fired Semirara and Indonesian coals; and tested the synthesized product for application as a detergent zeolite particularly as a potential surfactant carrier in laundry detergents as indicated by its liquid-carrying capacity.

\section{MATERIALS AND METHODS}

\section{A. Materials}

The study used the CFB fly ash from a coal mix of Semirara and Indonesian coal. This fly ash is available in the chemical engineering laboratory. Hydrochloric acid (B.E. Scientific) was used for the pre-treatment to remove metal impurities and increase silicon and aluminum ratio. The synthesis experiment used sodium hydroxide pellets (B.E. Scientific) for the fusion of fly ash. Distilled water was used as solvent.

Glass beakers $(250 \mathrm{ml})$ were used for all mixing and dissolution activities. Stainless steel containers were used as holder for the reagents for the fusion reaction inside the muffle furnace. Hot plate with magnetic stirrer along with medium sized spin bar were used for $\mathrm{NaOH}$ dispersal and aging. The gravity convection oven was used for crystallization.

\section{B. Design of Experiment}

A 3-level and 2-factor experimental design was chosen. Factors held constant were solvent (water), T-element source (CFB fly ash), mineralizer and template $(\mathrm{NaOH})$, fusion temperature $(823 \mathrm{~K})$, aging time (18 hours) and crystallization temperature $(363 \mathrm{~K})$. The concentration and $\mathrm{NaOH} /$ fly ash ratio will be varied according to Table I.

TABLE I: 3-LEVEL 2-FACTOR EXPERIMENTAL DESIGN

\begin{tabular}{|l|c|c|}
\hline Sample & HCl reflux concentration & $\begin{array}{c}\mathbf{N a O H / F l y ~ A s h ~} \\
\text { ratio }\end{array}$ \\
\hline S1 & $0 \mathrm{M}$ & 1.1 \\
\hline S2 & $3 \mathrm{M}$ & 1.1 \\
\hline S3 & $5 \mathrm{M}$ & 1.3 \\
\hline S4 & $3 \mathrm{M}$ & 1.3 \\
\hline S5 & $5 \mathrm{M}$ & 1.1 \\
\hline S6 & $0 \mathrm{M}$ & 1.3 \\
\hline
\end{tabular}

\section{Synthesis}

Prior to all steps, the fly ash obtained as is was first screened using Tyler Mesh sizes 30, 100, and 120. The oversize from Tyler-100 was kept and was used all throughout.

Ten (10) grams of raw fly ash was refluxed with $\mathrm{HCl}$ for one (1) hour. The refluxed fly ash was then filtered and dried. This was done in order to enhance stability of the framework and reduce metal contaminants by dissolution into ions [9].

The refluxed fly ash was mixed with $\mathrm{NaOH}$ and was boiled off until the sludge was dry. Dispersion ensures uniform concentration of $\mathrm{NaOH}$ in the fly ash and promotes good contact of reactants that would otherwise hinder the fusion reaction due to mass transfer limitations.

The sludge was then transferred into a stainless steel container and heated in a muffle furnace at $550^{\circ} \mathrm{C}$ for 1 hour. The fused solids were then crushed into powder and dissolved in $100 \mathrm{~mL}$ water. The slurry was mixed at $300 \mathrm{rpm}$ for 18 hours [10]. The slurry was filtered and the filtrate was kept at $90^{\circ} \mathrm{C}$ without disturbance [9]. The crystals formed were filtered, washed and dried.

\section{Characterization and Testing}

For the liquid-carrying capacity test, five (5) grams of the crystallized product was accurately weighed in a $50 \mathrm{~mL}$ beaker. Distilled water was added dropwise using a burette while stirring continuously with a glass rod. This was done until the entire mass agglomerated into a dough [11].

\section{RESULTS AND DISCUSSION}

\section{A. Synthesis}

Results from various testing show transformation of fly ash into zeolites. Fig. 1 shows the FTIR spectra of the fly ash, 
synthesized zeolite, and commercial zeolites. In general, the IR spectrum can be split into two groups of vibrations: (i) internal vibrations of framework $\mathrm{TO}_{4}$ units, which are insensitive to the structural vibrations; and (ii) vibrations related to the external linkage of the $\mathrm{TO}_{4}$ units in the structures [10]. Frequency regions common to all zeolites are summarized in Table II. Clearly, the FTIR results suggest the transformation of fly ash into zeolitic materials. Referring to Table II, the synthesized zeolite exhibited asymmetric stretching evident in the sharp $1000 \mathrm{~cm}^{-1}$ peak. The broad band on the fly ash spectrograph near this region intensified and formed the said peak exhibited by the synthesized material. This peak is also associated with $\mathrm{Si}-\mathrm{O}-\mathrm{Si}$ and Al-O-Al tetrahedral bonding vibrations that are present in all three materials with increasing intensities [12]. Another intense band characteristic of zeolites is the bending mode of $\mathrm{T}-\mathrm{O}$ at $430-500 \mathrm{~cm}^{-1}$. This however, was not exhibited by the synthesized fly ash zeolite.

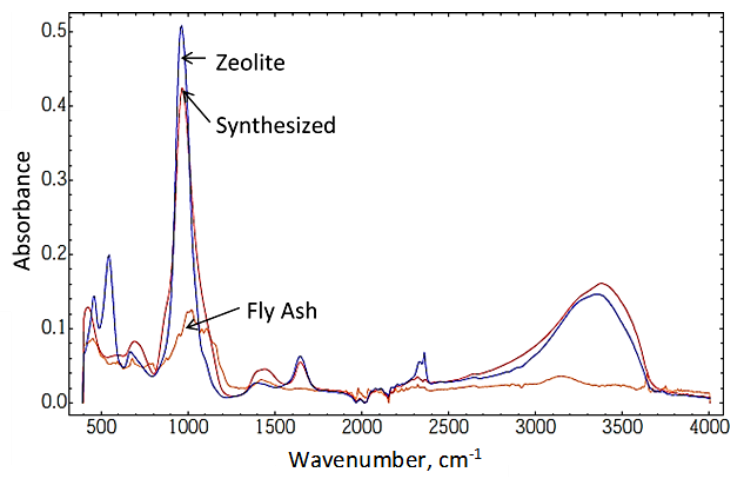

Fig. 1. IR Spectra of fly ash, synthesized and commercial zeolites.

TABLE II: ZEOLITE IR ASSIGNMENTS ${ }^{10}$

\begin{tabular}{|l|c|}
\hline \multicolumn{1}{|c|}{ Assignment } & Wavenumber, $\mathbf{~ m}^{\mathbf{- 1}}$ \\
\hline Asymmetric stretch (internal linkage) & $1250-950$ \\
\hline Symmetric stretch & $720-650$ \\
\hline T-O bend & $430-500$ \\
\hline Double ring & $650-500$ \\
\hline Pore opening & $300-420$ \\
\hline Asymmetric stretch (external linkage) & $100-1150$ (sharp) \\
\hline
\end{tabular}

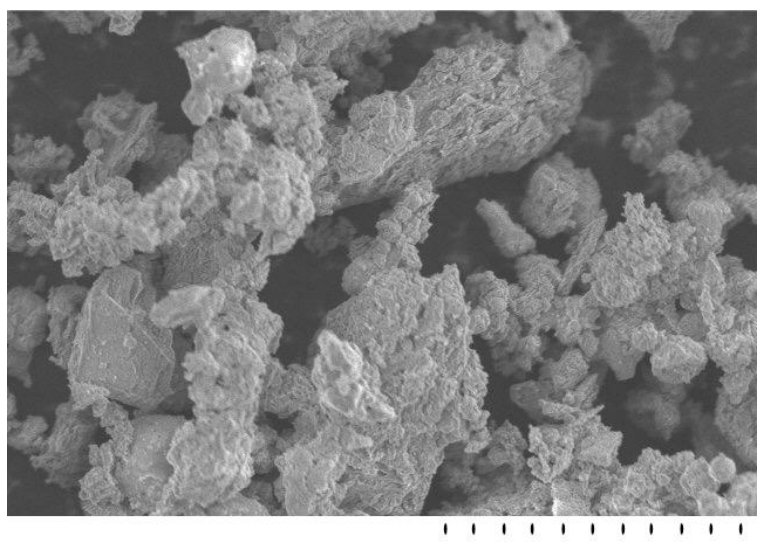

S3400 5.00KV $7.9 \mathrm{~mm} \times 1.00 \mathrm{~K}$ SE 4/2/2014 02:51 50.0um

Fig. 2. CFB fly ash SEM image at 1000x magnification.

SEM analysis is a powerful tool for observing the physical characteristics and changes in the materials. Fig. 2, Fig. 3 and Fig. 4 show the images produced by the analysis. The non-uniform shape and particle size of CFB fly ash is one of its distinguishing characteristics from that of a fly ash from PC boilers. The angular nature of these particles is attributed to the minerals present in fly ash as well as the significantly lower temperatures experienced by particles in the CFB boilers. Upon synthesis treatment, there is evident transformation into more uniform particles in terms of size and shape, particularly into a more rounded form.
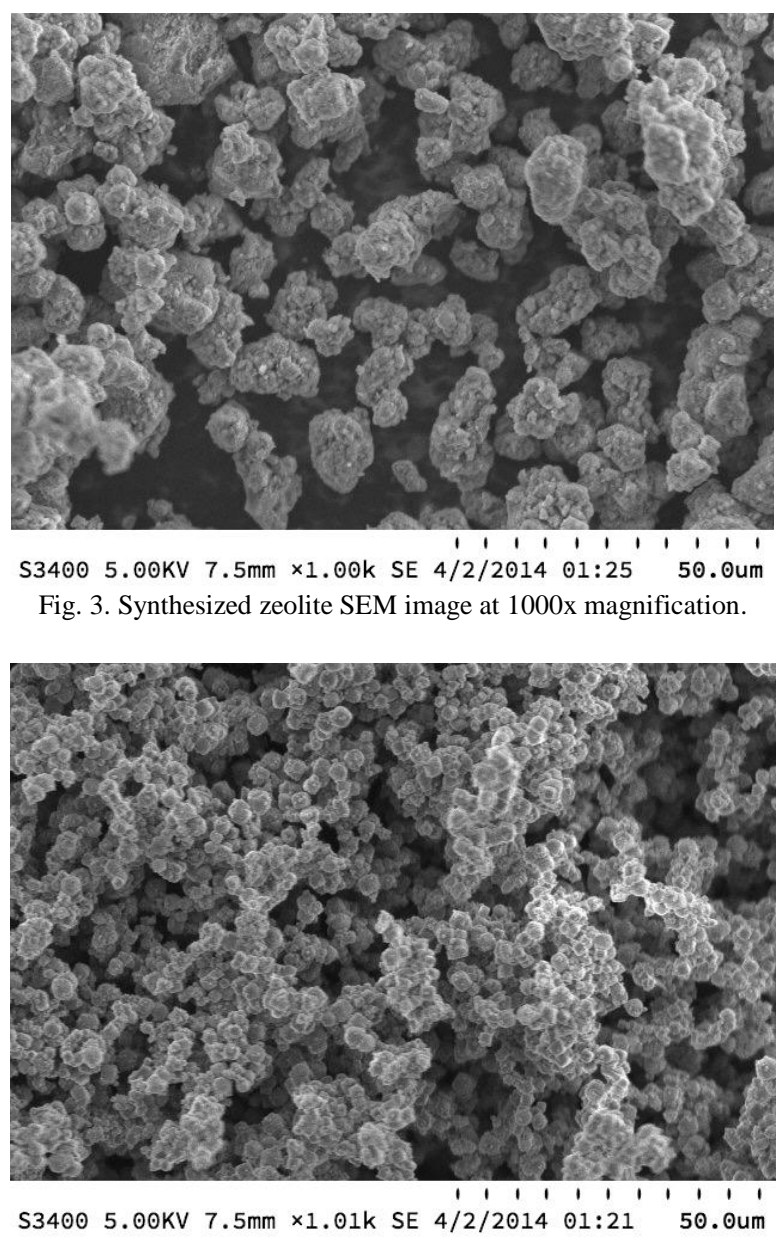

Fig. 4. Commercial zeolite SEM image at $1000 \mathrm{x}$ magnification.

Fig. 5 shows the synthesized and commercial zeolites at 5000x magnification. The images show that the characteristic octahedral crystal structure, as seen from the commercial zeolites, is not well formed in the synthesized zeolites. Particle sizes of the synthesized zeolites are also much larger compared to the commercial zeolites. The neat and flat face of each octahedral particle in the commercial zeolites is noticeably different from the coarse particles of the synthesized zeolites. This indicates that the component T-elements of the zeolites, albeit present in the system, did not crystallize well as expected.

Fig. 6 shows the XRD patterns of the raw fly ash and the synthesized zeolites. The synthesis treatment produced changes in material phases. The sharp peak at around 2 theta of 26 is representative of the quartz phase. The XRD patterns shown by Fig. 7 supports what is observed in the SEM analysis. Sharp diffraction peaks expected from zeolites were not present in the synthesized zeolites. The synthesized zeolites exhibited amorphous phase as opposed to crystalline phase. This is shown by the broad and wave-like shape that is present in the XRD pattern. 
The metastable gel phase was not readily observed in the solution, supporting the deprivation of free T-element components in solution insufficient for supersaturation.

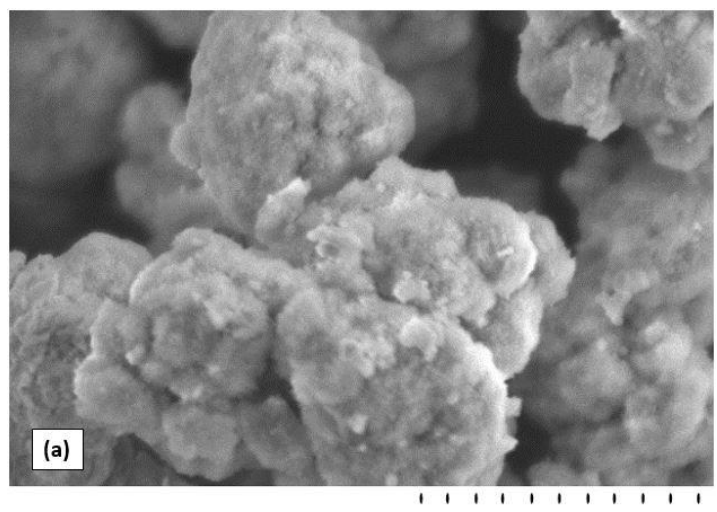

S3400 5.00KV $7.5 \mathrm{~mm} \times 5.00 \mathrm{~K}$ SE $4 / 2 / 2014 \quad 01: 28 \quad 10.0 \mathrm{um}$

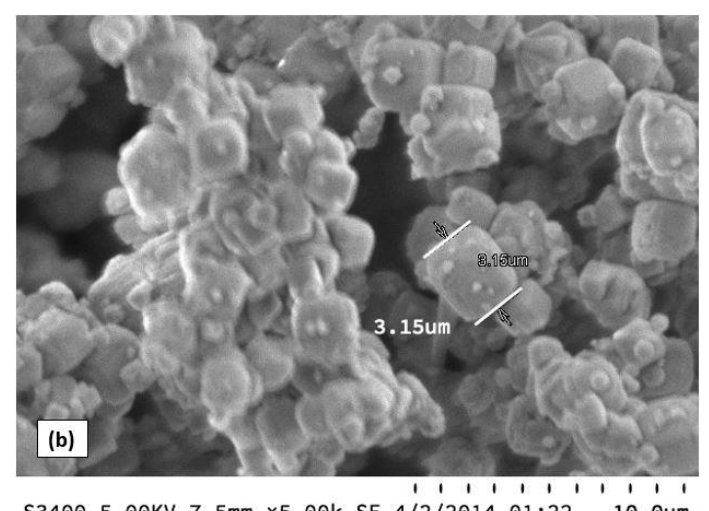

S3400 5.00KV $7.5 \mathrm{~mm} \times 5.00 \mathrm{k}$ SE $4 / 2 / 2014 \quad 01: 22 \quad 10.0 \mathrm{um}$

Fig. 5. Synthesized (a) and Commercial (b) zeolites at 5000x magnification.

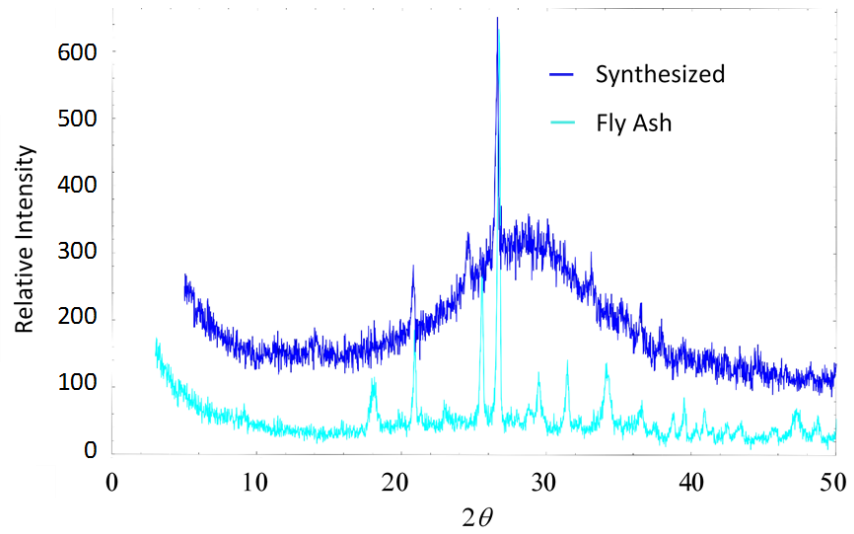

Fig. 6. XRD powder diffraction pattern of fly ash and synthesized zeolites.

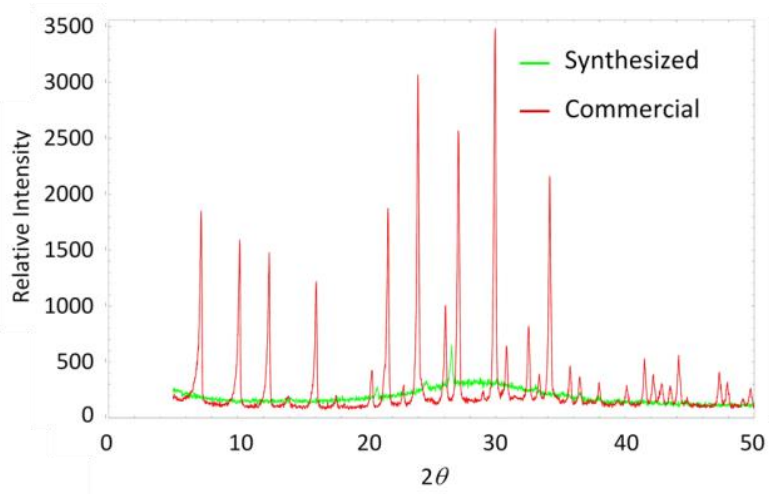

Fig. 7. XRD powder diffraction pattern of synthesized and commercial zeolites.

\section{B. Liquid-Carrying Capacity}

The next figure, Fig. 8, shows the results of the performance testing conducted on the synthesized samples. As can be seen, five synthesized zeolite samples perform better than the commercial zeolites in terms of liquid carrying capacity. High liquid carrying capacity suggests high absorbance of surfactants when added to the laundry detergents. Surfactants are the main components in the detergents that remove dirt present in clothes. This may indicate better performance as detergent additive. Analysis also shows that the concentration of $\mathrm{HCl}$ used in fly ash pre-treatment does not affect the performance of the synthesized zeolites as potential surfactant carriers.

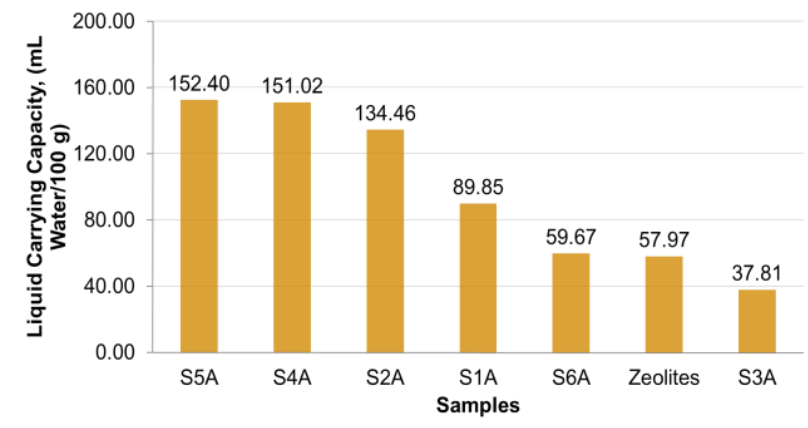

Fig. 8. Performance testing of synthesized zeolites.

\section{CONCLUSION AND RECOMMENDATIONS}

In line with the objectives of the study, the CFB fly ash from the coal mix of Semirara and Indonesian coals can be transformed into materials that are precursors to zeolites.

The method employed in the study suggests that it was successful in terms of product transformation. Certain impurities present in the sample may have interfered in the crystallization step, suppressing the formation of highly crystalline zeolitic materials. In terms of liquid carrying capacity, five out of the six samples of synthesized zeolites performed better than the commercial zeolite which indicates potential applicability to industry use.

The method employed successfully transformed fly ash into elements that are precursors to zeolites. FTIR Spectra has shown a match in wave numbers for commercial and synthesized zeolites.

The atomic arrangement of these elements, however, deviates from that of a zeolite, evident from particle shapes revealed by SEM analysis. The crystallinity of the synthesized zeolites also does not match that of the commercial zeolites based on XRD analysis.

$\mathrm{HCl}$ pretreatment does not affect the liquid carrying capacity. Fly ash to $\mathrm{NaOH}$ ratio however affects the liquid carrying capacity inversely. The liquid carrying capacity of Sample $6(0 \mathrm{M} / 1: 1.3)$ has the closest value to the commercial zeolites, but Sample $5(5 \mathrm{M} / 1: 1.1)$ has the largest liquid carrying capacity value, almost 3 times that of the commercial zeolites.

It is recommended to subject the sample to Atomic Absorption Spectroscopy analysis to determine the possible metal contaminants. Consider the use of hydrothermal crystallization in an autoclave. It is recommended to use a nickel crucible instead of stainless steel to mitigate suspected contamination evident from color differences in samples 
during aging. Washing of refluxed fly ash with distilled water until the supernatant is clear and colorless is also recommended before proceeding to further steps to assure removal of recontamination from adhering solution. The use of Teflon beaker or container for crystallization is highly recommended to eliminate the interference of scratches and coarse surface of glass material in nucleation. Further performance tests on the material as synthesized are needed to confirm performance as a detergent zeolite.

\section{ACKNOWLEDGMENT}

The authors thank the Department of Science and Techology Engineering Research and Development for Technology (DOST-ERDT) and the U.P. Engineering Research and Development Foundation, Inc. for assistance extended to the performance, dissemination and publication of this work.

\section{REFERENCES}

[1] M. Ahmaruzzaman, "A review on the utilization of fly ash," Progress in Energy and Combustion Science, vol. 36, no. 3, pp. 327-363, June 2010.

[2] I. Lecuyer, S. Gueraud, and J. M. Bursi, "Different uses of CFB ash-EDF investigations," International Energy Agency-Fluidized Bed Conversion, 1996.

[3] J. Hagen Industrial Catalysis: A Practical Approach., ch. 7, pp. 239-241, 2006

[4] J. L. Guth and H. Kessler, "Synthesis of aluminosilicate zeolites and related silica-based materials," in Catalysis and Zeolites: Fundamentals and Applications, J. Weitkamp and L. Puppe, Eds. Heidelberg, Springer-Verlag, 1999, pp. 18-23.

[5] H. Hayashi, K. Miyaura, and N. Shigemoto, "Selective formation of Na-X zeolite from coal fly ash by fusion with sodium hydroxide prior to hydrothermal reaction," J Mater Sci., vol. 28, pp. 4781-4786, 1993.

[6] K. Ojha, N. Pradhan, and A. Samanta, "Zeolite from fly ash: synthesis and characterization," Bull Mater Sci., vol. 27, no. 6, pp. 555-564, 2004.

[7] S. Sutarno and Y. Arryanto, "Synthesis of faujasite from fly ash and its applications for hydrocracking of petroleum distillates," Bull Chem React, vol. 2, pp. 45-51, 2007.

[8] N. Koukouzas and C. Vasilatos, "Characterization of CFB-coal fly ash zeolitic materials and their potential use in wastewater treatment,' presented at the 2009 World Coal Ash, Lexington, KY, USA, May 4-5, 2009.

[9] S. Sutarno and Y. Arryanto, "Synthesis of faujasite from fly ash and its applications for hydrocracking of petroleum distillates," Bull Chem React, vol. 2, pp. 45-51, 2007.

[10] K. Ojha, N. Pradhan, and A. Samanta, "Zeolite from fly ash: Synthesis and characterization," Bull Mater Sci., vol. 27, no. 6, pp. 555-564, 2004.
[11] UMA-HPC Method of Analysis, Unilever Philippines, Manila, MM, pp. 6-7.

[12] Tables of molecular vibrational frequencies consolidated volume 1. (Jun. 1972). Nat. Stand. Ref. Data Sen, Nat. Bur. Stand (U.S.). [Online]. http://www.nist.gov/data/nsrds/NSRDS-NBS-39.pdf Available:

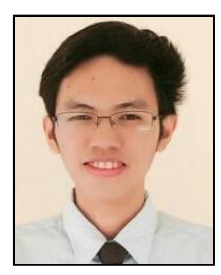

Jay V. Ranoco hails from Sogod, Southern Leyte, and earned his B.S. chemical engineering degree from the University of the Philippines, Diliman, Quezon City, Philippines in 2014, and is graduating magna cum laude.

$\mathrm{He}$ is currently an operations engineer at Petron Bataan Refinery in Limay, Bataan.

Engr. Ranoco is a member of the Philippine Institute of Chemical Engineers, Bataan Chapter. He was a national finalist in the Procter \& Gamble ASEAN Business Challenge in 2013 and was the Champion, along with his groupmate JC Quintos, in the Unilever Undergraduate Research Grant Competition in 2014.

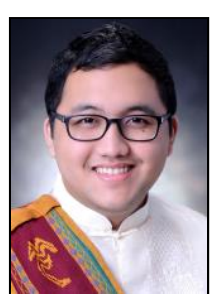

JC Justine G. Quintos earned his B.S. chemical engineering degree from the University of the Philippines, Diliman, Quezon City, Philippines in 2014 $\mathrm{He}$ is currently pursuing the M.S. in applied mathematics at the University of the Philippines, Diliman.

Engr. Quintos is a member Philippine Institute of Chemical Engineers and was co-awardee with Engr. Ranoco of the Unilever Undergraduate Research Grant given by Unilever Philippines in 2014.

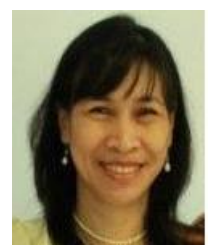

Rizalinda L. de Leon was born in Manila, Philippines and earned her B.S. chemical engineering, M.S. energy engineering, and $\mathrm{Ph} . \mathrm{D}$. chemical engineering degrees from the University of the Philippines, Diliman, Quezon City, Philippines in 1982, 1988, and 2007 , respectively.

She is currently Chair and Associate Professor at the Department of Chemical Engineering, College of Engineering, University of the Philippines, Diliman, Quezon City.

Dr. de Leon is president of the Philippine Institute of Chemical Engineers, Metro Manila Academe Chapter (PIChE-MMAC). She was awarded by PIChE as Outstanding Researcher during its 2015 national convention held in Puerto Princesa, Palawan. She also received the Chancellor's Award for Outstanding Teacher, given by the University of the Philippines, Diliman. She received the College of Engineering Outstanding Engineering Professor Award in 2012 and the College of Engineering Outstanding Researcher Award in 2015. She is recipient of the 2015 Dean Marino Mena Professorial Chair Award for her research accomplishments. 